\title{
Coefficient Inequalities for Uniformly P-Valent Starlike and Convex Functions
}

\section{Vandna Agnihotria ${ }^{1 *}$ and Ran Singh ${ }^{2}$}

${ }^{1}$ D-09, The LNM Institute of Information Technology, Jaipur-302031, Rajasthan, India

${ }^{2}$ Department of Mathematics, DAV College, CSJM University, Kanpur-208016, UP, India

\begin{abstract}
In this paper we obtain some coefficient inequalities for subclasses of uniformly $p$-valent starlike and convex functions in the open unit disk denoted by $\operatorname{SD}_{p}(\beta, \alpha)$ and $\operatorname{KD}_{p}(\beta, \alpha)$. Growth bounds and distortion bounds are discussed for functions in these classes. For different values of the parameters $p, \alpha$ and $\beta$ our results of this paper generalize those obtained by several authors in the literature.
\end{abstract}

Keywords: P-valent functions; Starlike functions; Convex functions

\section{Introduction}

Let $U=\{z \in \mathbb{C}:|z|<1\}$ denote the open unit disk and let $\mathrm{A}_{p}$ be the class of functions $\mathrm{f}(\mathrm{z})$ of the form $f(z)=z^{p}+\sum_{n=p+1}^{\infty} a_{n} z^{n}, p \in \mathbb{N}=\{1,2, \ldots\}$ which are analytic in the open unit disk $U$. A function $f \in A$ is said to be $\mathrm{p}$-valent starlike of order $\alpha(0 \leq a<\mathrm{p})$, if

$$
\mathfrak{R}\left(\frac{z f^{\prime}(z)}{f(z)}\right)>\alpha, \quad z \in U .
$$

The class of all such functions is denoted by $S_{p}^{*}(\alpha)$. A function $\mathrm{f}$ $\in \mathrm{A}_{\mathrm{p}}$ is said to be $\mathrm{p}$-valent convex of order $\alpha(0 \leq \alpha<\mathrm{p})$, if

$$
\Re\left(1+\frac{z f^{\prime \prime}(z)}{f^{\prime}(z)}\right)>\alpha, z \in U
$$

Let $K_{p}(\alpha)$ denote the class of all such functions. For $p=1$ we write $\mathrm{A} 1=\mathrm{A}$. Note that for $\mathrm{p}=1$ the classes $S_{1}^{*}(\alpha)$ and $\mathrm{K}_{1}(\alpha)$ are the usual classes of starlike and convex functions of order $\alpha(0 \leq \alpha<1)$ respectively, and will be denoted by $S^{*}(\alpha)$ and $K(\alpha)$ respectively. For $p=1$ and $\alpha=0$, the classes $S_{p}^{*}(\alpha)$ and $\mathrm{Kp}(\alpha)$ reduces to $\mathrm{S}^{*}(0)=\mathrm{S}^{*}$ and $\mathrm{K}(0)=\mathrm{K}$ respectively, which are the classes of starlike (with respect to the origin) and convex functions.

\section{The Subclasses $\mathrm{SD}_{\mathrm{p}}(\boldsymbol{\beta}, \boldsymbol{\alpha})$ and $\mathrm{KD}_{\mathrm{p}}(\boldsymbol{\beta}, \boldsymbol{\alpha})$}

We begin this Section by remark that this article is motivated by the work of Owa et al. [1]. We now recall the definitions of the subclasses $\mathrm{SD}_{\mathrm{p}}(\beta, \alpha)$ and $\mathrm{KD}_{\mathrm{p}}(\beta, \alpha)$ of uniformly $\mathrm{p}$-valent function introduced and studied by Agnihotri and Singh [2].

A function $\mathrm{f} \in \mathrm{Ap}$ is said to be in the class $\mathrm{SD}_{\mathrm{p}}(\beta, \alpha)$ if

$$
\Re\left(\frac{z f^{\prime}(z)}{f(z)}\right)>\beta\left|\frac{z f^{\prime}(z)}{f(z)}-p\right|+\alpha, z \in U,
$$

for some $\beta \geq 0$ and $\alpha(0 \leq \alpha<p)$.

A function $\mathrm{f} \in \mathrm{Ap}$ is said to be in the class $\operatorname{KDp}(\beta, \alpha)$ if

$$
\Re\left(1+\frac{z f^{\prime \prime}(z)}{f^{\prime}(z)}\right)>\beta\left|\frac{z f^{\prime \prime}(z)}{f^{\prime}(z)}-(p-1)\right|+\alpha, z \in U,
$$

for some $\beta \geq 0$ and $\alpha(0 \leq \alpha<p)$. Note that $f(z) \in \operatorname{KD}_{p}(\beta, \alpha)$ if and only if $\mathrm{zf}^{\prime}(\mathrm{z}) \in \mathrm{SD}_{\mathrm{p}}(\beta, \alpha)$. Agnihotri and Singh [2] have shown some sufficient conditions for $\mathrm{f}$ to be in the classes $\operatorname{SD}_{\mathrm{p}}(\beta, \alpha)$ and $\mathrm{KD}_{\mathrm{p}}(\beta, \alpha)$.
The subclasses $\operatorname{SD}_{1}(\beta, \alpha)$ and $\mathrm{KD}_{1}(\beta, \alpha)$ which will also be denoted by $\operatorname{SD}(\beta, \alpha)$ and $\operatorname{KD}(\beta, \alpha)$ respectively were studied by Shams, Kulkarni and Jahangiri in [3]. They have obtained sufficient conditions for $\mathrm{f}$ to be in the classes $\operatorname{SD}(\beta, \alpha)$ and $\mathrm{KD}(\beta, \alpha)$.

\section{Coefficient Inequalities}

We now give coefficient inequalities for functions belonging to the subclasses $\operatorname{SD}_{\mathrm{p}}(\beta, \alpha)$ and $\mathrm{KD}_{\mathrm{p}}(\beta, \alpha)$. Our first result is contained in

Theorem 3.1: If $f \in S D_{P}(\beta, \alpha)$ with $0 \leq p \beta \leq \alpha<p$, then $f \in s_{p}^{*}\left(\frac{\alpha-p \beta}{1-\beta}\right)$ and if $\beta>\frac{p+\alpha}{2 p}$ then $f \in s_{p}^{*}\left(\frac{\alpha-p \beta}{\beta-1}\right)$

Proof: We know that $\mathfrak{R}(\mathrm{z}) \leq|\mathrm{z}|$ for any complex number $\mathrm{z}$. Therefore $f \in S D_{p}(\beta, \alpha)$ gives us

$$
\Re\left(\frac{z f^{\prime}(z)}{f(z)}\right)>\beta \Re\left(\frac{z f^{\prime}(z)}{f(z)}-p\right)+\alpha
$$

From this we get

$$
\mathfrak{R}\left(\frac{z f^{\prime}(z)}{f(z)}\right)>\frac{\alpha-p \beta}{1-\beta}(z \in U) .
$$

Now, if $0 \leq p \beta \leq \alpha<p$, then it follows that

$$
0 \leq \frac{\alpha-p \beta}{1-\beta}<p
$$

and if $\beta>\frac{p+\alpha}{2 p}$, then we have

$$
-p<\frac{p \beta-\alpha}{\beta-1} \leq 0
$$

*Corresponding author: Vandna Agnihotria, D-09, The LNM Institute of Information Technology, Jaipur-302031, Rajasthan, India, E-mail: ifliitk02@gmail.com

Received July 16, 2013; Accepted October 15, 2013; Published October 21, 2013

Citation: Agnihotria V, Singh R (2013) Coefficient Inequalities for Uniformly P-Valent Starlike and Convex Functions. J Appl Computat Math 2: 143. doi:10.4172/2168-9679.1000143

Copyright: (c) 2013 Agnihotria V, et al. This is an open-access article distributed under the terms of the Creative Commons Attribution License, which permits unrestricted use, distribution, and reproduction in any medium, provided the original author and source are credited. 
Thus, $0 \leq \frac{\alpha-p \beta}{\beta-1}<p$,

For $\mathrm{p}=1$, we obtain the following corollary due to Owa, Polato`glu, and Yuvaz [1].

Corollary 3.1: If $\mathrm{f} \in \mathrm{SD}(\beta, \alpha)$ with $0 \leq p B \leq \alpha$ then $f \in S^{*}\left(\frac{\alpha-\beta}{1-\beta}\right)$.

Next, we state the corresponding result for functions belonging to the subclass $K D_{p}(\beta, \alpha)$.

Theorem 3.2: If $f \in K D_{p}(\beta, \alpha)$ with $0 \leq p B \leq \alpha<p$ then $f \in K_{p}\left(\frac{\alpha-p \beta}{1-\beta}\right)$ and if $\beta>\frac{p+\alpha}{2 p}$, then $f \in K_{p}\left(\frac{\alpha-p \beta}{\beta-1}\right)$

Proof: Proof is similar to the proof of Theorem 3.1.

The following corollary is due to Owa, Polato glu, and Yuvaz [1] for $\mathrm{p}=1$.

Corollary 3.2: If $f \in K D(\beta, \alpha)$ with $0 \leq \beta \leq \alpha$ then $f \in K\left(\frac{\alpha-\beta}{1-\beta}\right)$ We now state the main theorem of this paper.

Theorem 3.3: If $f \in S D_{p}(\beta, \alpha)$ then $\left|a_{p}+1\right| \leq \frac{2(p-\alpha)}{|1-\beta|}$

and $\left|a_{p}+n\right| \leq \frac{2(p-\alpha)}{n|1-\beta|} \prod_{j=1}^{n-1}\left(1+\frac{2(p-\alpha)}{j|1-\beta|}\right)(n \geq 2)$

Proof: We know that if $f \in S D_{p}(\beta, \alpha)$, then $R\left(\frac{z f^{\prime}(z)}{f(z)}\right)>\frac{\alpha-p \beta}{1-\beta}(z \in U)$

Define a function $\mathrm{q}(\mathrm{z})$ by

$$
q(z)=\frac{(1-\beta)\left(\frac{z f^{\prime}(z)}{f(z)}\right)-(\alpha-p \beta)}{(p-\alpha)}(z \in U)
$$

Note that $\mathrm{q}$ is analytic in $\mathrm{U}$ with $q(0)=1$ and $R(q(z))>0$ If $q(z)=1+q_{1} z+q_{2} z^{2}+\ldots$,

then we can write $\frac{z f^{\prime}(z)}{f(z)}=\frac{\alpha-p \beta}{1-\beta}+\frac{p-\alpha}{1-\beta} \sum_{n=0}^{\infty} q_{n} z^{n}$

or $z f^{\prime}(z)=f(z)\left(p+\left(\frac{p-\alpha}{1-\beta}\right) \sum_{n=1}^{\infty} q_{n} z^{n}\right),\left(q_{0}=1\right)$.

From this, we obtain

$$
n a_{p+n}=\left(\frac{p-\alpha}{1-\beta}\right)\left(q_{n}+a_{p}+1 q_{n-1}+a_{p-} 2 q_{n-2}+\ldots+a_{p}+n-1 q_{1}\right)
$$

From the coefficient estimates for Carath' eodory functions [4], we know that $\left|q_{n}\right| \leq 2$ for all $n \geq 1$

Making use of it in (3.7) we see that

$$
\left|a_{p+n}\right| \leq \frac{2(p-\alpha)}{n|1-\beta|}\left(1+\left|a_{p+1}\right|+\left|a_{p+2}\right|+\ldots+\left|a_{p+n-1}\right|\right) \text {. }
$$

Therefore, for $\mathrm{n}=1$, we have

$$
\left|a_{p+1}\right| \leq \frac{2(p-\alpha)}{|1-\beta|}
$$

which proves (3.3). Now for $\mathrm{n}=2$, we obtain $\left|a_{p+2}\right| \leq \frac{2(p-\alpha)}{2|1-\beta|},\left(1+\left|a_{p+1}\right|\right)$ $\leq \frac{2(p-\alpha)}{2|1-\beta|}\left(1+\frac{2(p-\alpha)}{|1-\beta|}\right)$.

This shows that (3.4) is true for $n=2$. For $n=3$, we see that

$$
\left|a_{p+3}\right| \leq \frac{2(p-\alpha)}{3|1-\beta|}\left(1+\left|a_{p+1}\right|+\left|a_{p+2}\right|\right) \quad \leq \frac{2(p-\alpha)}{3|1-\beta|}\left(1+\frac{2(p-\alpha)}{|1-\beta|}+\frac{2(p-\alpha)}{2|1-\beta|}+\frac{2^{2}(p-\alpha)^{2}}{2|1-\beta|^{2}}\right)
$$

Thus, (3.4) holds for $\mathrm{n}=3$. Next, we assume that (3.4) is true for $\mathrm{n}=\mathrm{k}$ and therefore

$$
\begin{aligned}
& \left|a_{p+k+1}\right| \leq \frac{2(p-\alpha)}{(k+1)|1-\beta|}\left(1+\frac{2(p-\alpha)}{|1-\beta|}+\frac{2(p-\alpha)}{|1-\beta|}\left(1+\frac{2(p-\alpha)}{|1-\beta|}\right)+\ldots \frac{2(p-\alpha)}{k|1-\beta|} \prod_{j=1}^{k-2}\left(1+\frac{2(p-\alpha)}{j|1-\beta|}\right)\right) \\
& \leq \frac{2(p-\alpha)}{(k+1)|1-\beta|} \prod_{j=1}^{k-1}\left(1+\frac{2(p-\alpha)}{j|1-\beta|}\right) .
\end{aligned}
$$

This shows that (3.4) is true for $\mathrm{n}=\mathrm{k}+1$. Hence, by using the principle of mathematical induction, (3.4) holds for all $\mathrm{n} \geq 2$.

Remark 3.1: Taking $\mathrm{p}=1$ in Theorem 3.3 , we obtain

$$
\left|a_{p+1}\right| \leq \frac{2(1-\alpha)}{n|1-\beta|} \prod_{j=1}^{n-1}\left(1+\frac{2(p-\alpha)}{j|1-\beta|}\right)(n \geq 2)
$$

which was given by Owa, Polato ${ }^{2}$ glu and Yavuz [1]

Remark 3.2: Taking $p=1$ and $\beta=0$ in Theorem 3.3, we have

$\left|a_{n+1}\right| \leq \frac{1}{n !} \prod_{j=2}^{n+1}(j-2 \alpha) \quad(n \geq 1)$, which was proven by Robertson [5].

We know that $f \in K D_{p}(\beta, \alpha)$ if and only if $z f^{\prime} \in S D_{p}(\beta, \alpha)$ [2]. Thus, we have

Theorem 3.4: If $f(z) \in K D_{p}(\beta, \alpha)$ then

$$
\left|a_{p+1}\right| \leq \frac{2 p(p-\alpha)}{(p+1)|1-\beta|}
$$

and $\left|a_{p+1}\right| \leq \frac{2 p(p-\alpha)}{(p+1)|1-\beta|} \prod_{j=1}^{n-1}\left(1+\frac{2(p-\alpha)}{j|1-\beta|}\right)(n \geq 2)$.

Proof: For $f \in K D_{p}(\beta, \alpha)$ we know $z f^{\prime}(z)+p z^{p-1}+\sum_{n=p+1}^{\infty} n a_{n} z^{n-1} \in S D_{p}(\beta, \alpha)$. Therefore

$$
\mathfrak{R}\left(1+\frac{z f^{\prime \prime}(z)}{f^{\prime}(z)}\right)>\frac{\alpha-p \beta}{1-\beta}(z \in U) .
$$

Define a function $\mathrm{r}(\mathrm{z})$ by

$$
r(z) \frac{(1-\beta)\left(\frac{z f^{\prime \prime}(z)}{f^{\prime}(z)}\right)-(\alpha-p \beta)}{(p-\alpha)}(z \in U)
$$

Note that $\mathrm{r}$ is analytic in $\mathrm{U}$ with $\mathrm{r}(0)=1$ and $\Re(r(z))>0$ If $r(z)=1+q_{1} z+q_{2} z^{2}+\ldots$, then we can write

$$
\begin{aligned}
& 1+\frac{z f^{\prime \prime}(z)}{f^{\prime}(z)}=\frac{\alpha-p \beta}{1-\beta}+\left(\frac{p-\alpha}{1-\beta}\right) \sum_{n=0}^{\infty} r_{n} z^{n}, \text { or } \\
& z f^{\prime \prime}(z)=f^{\prime}(z)\left(p-1+\left(\frac{p-\alpha}{1-\beta}\right) \sum_{n=1}^{\infty} r_{n} z^{n}\right),\left(r_{0}=1\right) .
\end{aligned}
$$

From this, we obtain

$n(p+n) a_{p+n}=\left(\frac{p-\alpha}{1-\beta}\right)\left(p r_{n}+(p+1) a_{p+1} r_{n-1}+(p+2) a_{p+2} r_{n-2}+\ldots+(p+n-1) a_{p+n-1} r_{1}\right)$.

From the coefficient estimates for Carath' eodory functions [4] , we know that $\left|\mathrm{r}_{\mathrm{n}}\right| \leq 2$ for all $\mathrm{n} \geq 1$.

Making use of it in (3.16) we see that 
$\left|a_{p+n}\right| \leq \frac{2(p-\alpha)}{n(p+n)|1+\beta|}(p+(p+1))\left|a_{p+1}\right|+(p+2)\left|a_{p+1}\right|+\ldots+(p+n-1)\left|a_{p+n-1}\right|$

Therefore, for $\mathrm{n}=1$, we have

$$
\left|a_{p+1}\right| \leq \frac{2 p(p-\alpha)}{(p+1)|1-\beta|},
$$

which proves (3.12). Now for $\mathrm{n}=2$, we obtain

$$
\begin{aligned}
& \left|a_{p+2}\right| \leq \frac{2(p-\alpha)}{2(p+2)|1-\beta|}\left(p+(p+1)\left|a_{p+1}\right|\right. \\
& \left|a_{p+3}\right| \leq \frac{2(p-\alpha)}{3(p+3)|1-\beta|}\left(p+(p+1)\left|a_{p+1}\right|+(p+2)\left|a_{p+2}\right|\right) \\
& \leq \frac{2 p(p-\alpha)}{2(p+2)|1-\beta|}\left(1+\frac{2(p-\alpha)}{|1-\beta|}\right)
\end{aligned}
$$

This shows that (3.12) is true for $n=2$. For $n=3$, we see that

$$
\begin{aligned}
& \left|a_{p+3}\right| \leq \frac{2(p-\alpha)}{3(p+3)|1-\beta|}\left(p+(p+1)\left|a_{p+1}\right|+(p+2)\left|a_{p+2}\right|\right) \\
& \leq \frac{2(p-\alpha)}{3(p+3)|1-\beta|}\left(1+\frac{2(p-\alpha)}{|1-\beta|}+\frac{2(p-\alpha)}{2|1-\beta|}+\frac{2^{2}(p-\alpha)^{2}}{|1-\beta|^{2}}\right)
\end{aligned}
$$

Thus, (3.12) holds for $\mathrm{n}=3$. Next, we assume that (3.12) is true for $\mathrm{n}=\mathrm{k}$ and therefore

$$
\begin{aligned}
& \left|a_{p+k+1}\right| \leq \frac{2 p(p-\alpha)}{(k+1)(p+k+1)|1-\beta|}\left(1+\frac{2(p-\alpha)}{|1-\beta|}+\frac{2(p-\alpha)}{2|1-\beta|}\left(1+\frac{2(p-\alpha)}{|1-\beta|}\right)+\ldots\right. \\
& +\frac{2(p-\alpha)}{k|1-\beta|} \prod_{j=1}^{k-2}\left(1+\frac{2(p-\alpha)}{j|1-\beta|}\right) \\
& \leq \frac{2 p(p-\alpha)}{(k+1)(p+k+1)|1-\beta|} \prod\left(+\frac{2(p-\alpha)}{j|1-\beta|}\right)
\end{aligned}
$$

This shows that (3.12) is true for $\mathrm{n}=\mathrm{k}+1$. Hence, by using the principle of mathematical induction, (3.12) holds for all $n \geq 2$.

Remark 3.3: If we take $\mathrm{p}=1$ in Theorem 3.4, then

$\left|a_{n+1}\right| \leq \frac{2(1-\alpha)}{(n+1) n|1-\beta|} \prod_{j=1}^{n-1}\left(1+\frac{2(p-\alpha)}{j|1-\beta|}\right)(n \geq 2)$ which was proven by Owa et al. [1].

Remark 3.4: Taking $p=1$ and $\beta=0$ in Theorem 3.4, we get

$|a| \leq \frac{1}{(1) !} \prod(j-2)(n \geq 1), \quad$ which was proven by Robertson [5]

Theorem 3.5: If $f \in S D_{p}(\beta, \alpha)$

$$
\left.\max \left\{0,|z|^{p}-\frac{2(p-\alpha)}{|1-\beta|}|z|^{p+1}-\sum_{n=2}^{\infty} \frac{2(p-\alpha)}{n|1-\beta|}\left(\prod_{j=1}^{n-1} \frac{2(p-\alpha)}{j|1-\beta|}\right)\right)|z|^{p+n}\right\} \leq|f(z)|
$$

$$
\leq|z|^{p}+\frac{2(p-\alpha)}{|1-\beta|}|z|^{p+1} \sum_{n=2}^{\infty} \frac{2(p-\alpha)}{n|1-\beta|}\left(\prod_{j=1}^{n-1}\left(\frac{2(p-\alpha)}{j|1-\beta|}\right)\right)|z|^{p+n}
$$

and $\max \left\{0, p|z|^{p-1}-\frac{2(p+1)(p-\alpha)}{|1-\beta|}|z|^{p}-\sum_{n=2}^{\infty} \frac{2(p+n)(p-\alpha)}{n|1-\beta|}\left(\prod_{j=1}^{n-1}\left(1+\frac{2(p-\alpha)}{j|1-\beta|}\right)\right)\right.$

$$
\left.\times|z|^{p+n-1}\right\}\left|f^{\prime}(z)\right| \leq p|z|^{p-1}+\frac{2(p+1)(p-\alpha)}{|1-\beta|}|z|^{p}
$$

Proof: Proof follows from the fact that $f(z)=z^{p}+\sum_{k=p+1}^{\infty} a_{k} z^{k}, p=1,2, \ldots$ and using Theorem 3.3.

Corollary 3.3: If $f \in K D_{p}(\beta, \alpha)$ then

$$
\begin{aligned}
& \max \left\{0,|z|^{p}-\frac{2 p(p-\alpha)}{(p+1)|1-\beta|}|z|^{p+1}-\sum_{n=2}^{\infty} \frac{2 p(p-\alpha)}{n(p+n)|1-\beta|}\left(\prod_{j=1}^{n-1}\left(1+\frac{2(p-\alpha)}{j|1-\beta|}\right)\right)|z|^{p+n}\right\} \leq|f(z)| \\
& \leq|z|^{p}+\frac{2 p(p-\alpha)}{(p+1)|1-\beta|}|z|^{p+1}+\sum_{n=2}^{\infty} \frac{2 p(p-\alpha)}{n(p+n)|1-\beta|}\left(\prod_{j=1}^{n-1}\left(1+\frac{2(p-\alpha)}{j|1-\beta|}\right)\right)|z|^{p+n}
\end{aligned}
$$

and $\max \left\{0, p|z|^{p-1}-\frac{2 p(p-\alpha)}{|1-\beta|}|z|^{p}-\sum_{n=2}^{\infty} \frac{2 p(p-\alpha)}{n|1-\beta|}\left(\prod_{j=1}^{n-1}\left(1+\frac{2(p-\alpha)}{j|1-\beta|}\right)\right)|z|^{p+n-1}\right\} \leq\left|f^{\prime}(z)\right|$

$$
\left.\times|z|^{p+n-1}\right\}\left|f^{\prime}(z)\right| \leq p|z|^{p-1}+\frac{2(p+1)(p-\alpha)}{|1-\beta|}|z|^{p}
$$

Proof: Proof follows from the fact that

$$
f(z)=z^{p}+\sum_{k=p+1}^{\infty} a_{k} z^{k}, p=1,2, \ldots
$$

\section{References}

1. Owa S, Polatǒglu Y, Yavuz E (2006) Coefficient inequalities for classes of uniformly starlike and convex functions. J Ineq Pure and Appl Math 7.

2. Agnihotri $\mathrm{V}$, Singh $\mathrm{R}$ New subclasses of uniformly $p$-valent starlike and convex functions, to appear.

3. Shams S, Kulkarni SR, Jahangiri JM (2004) Classes of uniformly starlike and convex functions. Internat J Math Sci 55: 2959-2961.

4. Carath'eodory C (1911) "Uber den variabilit"atsbereich der Fourierschen konstanten von possitiven harmonischen funktionen. Rend Circ Palermo 32 193-217.

5. Robertson MS (1936) On the theory of univalent functions. Ann Math 37: 374-408 